\title{
EDUCABILIDAD Y ENSEÑABILIDAD EN LA EDUCACION FISICA, LA RECREACION, EL DEPORTE
}

\author{
Contreras, Fabian ${ }^{1}$, Ramirez Yamile ${ }^{2}$. \\ 1 Doctor en Ciencias de la Cultura Fisica, Docente Universidad del Atlantico Grupo de \\ Investigacion GREDFICAD, fabiancontreras@dcc.uniatlantico.edu.co \\ 2 Magister en Innovaciones Educativas, Programa Todos a Aprender, \\ yamileramirez1702@hotmail.com
}

\section{RESUMEN}

Abordar la enseñabilidad y la educabilidad como temas paralelos, en un contexto expositivo, no es del todo recomendable si se tiene en cuenta que cada una de ellas supone procesos de desarrollo completamente independientes. Pero, que en el contexto práctico ambos se relacionan y complementan, por ejemplificar, la educabilidad abre camino y cimenta la enseñabilidad, además, una y otra enfocan como objeto de estudio al ser humano garantizándole el proceso de aprendizaje durante toda su vida, el cual hace propio, de su personalidad e intelectualidad.

Centrar la atención en las condiciones de educabilidad de los adolescentes y adultos lleva a interrogar a la Universidad y específicamente a los programas de Educacion Fisica, Recreacion y Deportes respecto a qué es lo que espera de ellos. Estas condiciones no se definen en sí mismas, sino que resultan del modelo de alumno que presupone la institución de educación superior.

Palabras Claves: educabilidad, enseñabilidad, educación física, recreación, deportes, modelo pedagógico, educación superior

\section{ABSTRACT}

Addressing teachability and educabilidad as parallel themes in an exhibition context, it is not entirely advisable if you consider that each of them involves completely independent development processes. But in the practical context both relate and complement, for exemplifying the educabilidad open way and builds teachability also again and focus as an object of study the human being guaranteeing the learning process throughout their lives, which He makes himself, his personality and intellectuality .

Focus on the conditions for education of adolescents and adults leads to interrogate the University and specifically programs Physical Education, Recreation and Sports regarding what is waiting for them. These terms are not defined in themselves, but are student model presupposes the institution of higher education.

Keywords: educability, teachability, physical education, recreation, sports, educational model, higher education. 
Es pertinente acudir al hombre como el eje sobre el cual giran ambas posibilidades. Este ha de verse como un ser integral, con sensibilidad, con una gran necesidad de relación e interacción con otros seres que lo lleven a su realización como humano dentro de un contexto social, todo a través de la comunicación y ésta gracias a la educación, entendida como el núcleo de las relaciones entre costumbres y cambios de una sociedad (Cox, Cristian. 1998), orientando los desarrollos futuros de la condición humana, por tanto, es necesario que se le brinden espacios de formación y transformación del conocimiento. De este modo, la educabilidad y la enseñabilidad deben darle un lugar apropiado a los saberes provenientes de las ciencias que alimentan la reflexión pedagógica poniendo de presente el carácter inter y transdisciplinario de la educación.

Etimológicamente el término educabilidad se compone de dos vocablos, educa (educable, educación, del lat. Educāre), formación, acompañamiento, desarrollo y bilidad derivado del sufijo -dad, (cuando dad se aplica a adjetivos verbales terminados en -ble, se forman derivados terminados en -bilidad), proveniente del lat. -tas, -ātis, que significa 'cualidad', posibilidad de llegar a, potencialidad.

Comprendiéndose entonces, desde tiempos de Herbart, quien fue el primero en utilizar esta expresión, como ductilidad y plasticidad y concepto fundamental de la pedagogía, lo cual ha servido como base para los distintos planteamientos que se han generado al respecto. Hoy, a través de los postulados de Paciano Fermoso se define como la posibilidad y categoría humana. Una posibilidad, porque significa la viabilidad del proceso educativo y la afirmación de que la educación es factible; una categoría humana, por cuando se predica del hombre esta cualidad. Del hombre se dice que es sociable, histórico, perfectible y educable. Entre todas las categorías humanas la educabilidad ocupa un lugar privilegiado y hasta es, para muchos, la más esencial de todas ellas. La educabilidad es la condición primordial del proceso educativo.

En cuanto al concepto de enseñabilidad, del vocablo enseña (enseñable, del lat. insignīa, y plural neutro de insignis, que se distingue por alguna señal), que significa insignia, estandarte y señal y bilidad de cualidad. Como en el caso anterior, se refiere a la potencialidad que tienen las ciencias de informar, instruir sus saberes al sujeto, es decir, la posibilidad de ser transmitidas 0 enseñadas de acuerdo con los métodos y técnicas de su construcción original.

La enseñabilidad supone, según Fermoso, hablar de comunicabilidad, ésta ha de convertirse en la vía de acceso a los diversos saberes, pues si la enseñabilidad no desarrolla códigos elaborados (símbolos, estructuras sociolingüísticas formales), se puede desvirtuar de su finalidad, posiblemente al hecho que al transmitir oralmente inciden distintos factores, el grupo social, la formación académica hasta el mismo lenguaje personal y otros más. En otras palabras, debe existir un equilibrio en las manifestaciones lingüísticas tanto filosóficas como científicas del que enseña con respecto a la preparación del que aprende, el receptor. De la misma forma pueden resultar inadecuados los medios de la comunicación, que en el caso de los saberes son los métodos y las técnicas didácticas. Estos aspectos pueden limitar el objeto y el contenido de los saberes, pero, no deben coartar el gesto, la expresión, la palabra con sentido y lógica de los que aprenden.

En un sentido más amplio puede entenderse la educación en el hombre como el proceso en el que se genera, desarrollan, transforman, elaboran y construyen competencias especializadas, las cuales le permiten interactuar con los saberes en un contexto determinado.

Son varios los autores que atribuyen a Herbart tanto la introducción del término 
"educabilidad" como la situación del mismo en el conjunto de términos desde los que se articula el espacio nocional teórico de la educación .

"El concepto fundamental de la pedagogía es la educabilidad del alumno (...). El concepto de educabilidad (ductilidad, plasticidad) es de más basta extensión. Se extiende casi hasta los elementos de la materia.(...).De la educabilidad volitiva se encuentran rastros en las almas de los animales más nobles. Pero la educabilidad de la voluntad para la moralidad sólo la reconocemos en los hombres" (HERBART).En este párrafo alude Herbart con el término educabilidad a tres aspectos: la plasticidad, la evolución animal y la relación exclusiva al hombre (categoría humana).

A la educabilidad, se le ha denominado también como principio o límite de la educación, porque tiene sentido hablar de educabilidad con referencia a las fronteras humanas ya que la mismidad humana radica en la capacidad para reflexionar sobre sus fronteras y en consecuencia asumirlas y desarrollarlas. GARCIA CARRASCO: "Los determinantes de la naturaleza humana son sus límites fronterizos: el biopsíquico, el social y el espiritual, con sus genuinas traducciones en el mundo de la cultura, que por separado, pero, sobre todo, a través de actuaciones conjuntas e interrelacionadas definen el espacio de la educabilidad".

Centrar la atención en las condiciones de educabilidad de los adolescentes y adultos lleva a interrogar a la Universidad y específicamente a los programas de Educacion Fisica, Recreacion y Deportes respecto a qué es lo que espera de ellos. Estas condiciones no se definen en sí mismas, sino que resultan del modelo de alumno que presupone la institución de educación superior. ¿Cuál es el tipo de alumno que está en condiciones de responder a la dinámica que el sistema propone, y terminar exitosamente su carrera educativa? ¿En qué alumno están pensando los sistemas educativos cuando diseñan sus estrategias pedagógicas?. La noción de educabilidad debe ser comprendida como un concepto relacional, en tanto se define en la tensión entre los recursos que el estudiante porta y los que la universidad espera de ellos o exige. Es en esa relación, en el punto límite del encuentro entre estas dos esferas, donde se definen los criterios de educabilidad. "El estudiante de Educacion Fisica, Recreacion y Deportes está en la encrucijada de estas dos socializaciones, y el éxito universitario de unos se debe a la proximidad de estas dos culturas, la familiar y la institución de eduacion superior, mientras que el fracaso de otros se explica por las distancias de esas culturas y por el dominio social de la segunda sobre la primera" (Dubet y Martucelli, 2000). En la misma línea, Pierre Bourdieu señala que la productividad específica del trabajo especifico de la formacion universitaria se mide según el grado en que el sistema de los medios necesarios para el cumplimiento del trabajo pedagógico está objetivamente organizado en función de la distancia existente entre el habitus que pretende inculcar y el habitus producido por los trabajos pedagógicos anteriores (Tenti, 1994).

El hombre no se puede comprender como un ser estático, sino como un ser que proyecta hacia el futuro, y por esta razón toma como instrumento sus propias habilidades, las cuales va desarrollando desde su formación, permitiéndole llegar a ser un sujeto situado desde un conjunto de normas y valores, los cuales él no elige, pero que si debe determinarse y que busca querer ser feliz, lo cual significa realizarse desde su propio ser y desde su realización personal.

Es en este ámbito educativo que la educabilidad y la enseñabilidad cumple un papel estelar al inculcar a los sujetos de la comunidad, que la libertad de cada individuo 
solo puede llegar hacia una educación que prepara y habilita al ser humano para la vida en sociedad, a reconstruir una cultura que reconozca y valore a cada uno de los miembros como sujetos plenos de derecho, capacitando y formando lideres que abren oportunidades y espacios políticos, especialmente en las democracias, el cual será fruto de un sistema económico social y cultural.

\section{LAS CARACTERISTICAS DE EDUCABILIDAD YENSEÑABILIDAD}

LA

Hay por lo menos cinco aspectos que hacen posible la realización de la educabilidad, según Paciano Fermoso:

\section{- La educabilidad es personal:}

Es decir, es una exigencia individual inalienable e irrenunciable, que surge del manantial de la personalidad y la hominidad.

\section{La educabilidad es intencional:}

Es decir, la posibilidad no está a merced de unas leyes naturales, sino que el sujeto es dueño de sí mismo, y dirige el sesgo y el viaje, de acuerdo con unas metas o ideales que se autofija.

\section{La educabilidad es referencial:}

Porque no es una fuerza ciega, sino una capacidad ilustrada, que sabe hacia dónde se dirige; en otras palabras, es teleológica.

\section{La educabilidad es dinámica:}

Pues la realización del programa existencial de cada hombre supone actividad y dotación de potencialidades que buscan pasar el acto.

La educabilidad es necesaria:

Pues sin ella el hombre se vería privado de posibilidades de auto realización, de personalización y de socialización.

\section{Características de Enseñabilidad:}

Interviene activamente dos actores, el docente y su estudiante, y que cada uno de ellos es producto de circunstancias especificas que constituyen su realidad social su formación y experiencia.

"De este modo se realiza la aspiración de T. Herbart de que el maestro debe saber tanto de Pedagogía como de la ciencia que enseña. El diseño didáctico de una disciplina requiere en la actualidad fundamentarse en al menos tres dominios de conocimiento. Tomado del documento (La Educabilidad y La Enseñabilidad).

Condiciones de enseñabilidad de cada disciplina.

\section{Enfoque o teoría pedagógica.}

Identificación y descripción de las condiciones psicosocioculturales del alumno respecto del aprendizaje de la materia. El resultado de conjugar estos tres dominios, produce el diseño de la enseñanza que incluye la construcción de objetos de enseñanza y el plan didáctico de ejecución de la misma".

Esta perspectiva la educabilidad es la base fundamental en el rol del docente, puesto que es la posibilidad que tiene cada ciencia o área de ser enseñada de acuerdo con los contenidos y temáticas.

Por lo tanto es importante insistir que la enseñabilidad pase a centrarse en la potencializacion de los procesos de los estudiantes, de sus capacidades y destrezas y lo más importante que el alumno aprenda a aprender desde sus propias necesidades e intereses al conocimiento. 
Según el autor Hans-Georg Gadamer "Afirmo que la educación es educarse, que la formación es formarse. Con ello dejo conscientemente al margen los que puedan ser, obviamente, los problemas entre la juventud y sus preceptores, maestros 0 padres".

Para construir la base de educación, se debe empezar desde el contexto familiar, ya que los miembros que la conforman tienen la tarea de educar al niño, partiendo desde la comunicación, aprendiendo las normas de comportamiento, valores morales, enseñándole al estudiante de Educacion Fisica, Recreacion y Deportes que se debe hacer y que no se debe hacer, empleándose como punto de partida la diversidad desde la personalidad de cada individuo, y es en este espacio familiar que prepara al adolescente y adulto para las relaciones sociales y así mismo este identidad puede variar por las condiciones culturales donde se desenvuelve.

Según John Dewey el sentido social de la educación es "Resultado neto obtenido hasta ahora, es que el ambiente social forma la disposición mental y emocional de la conducta en los individuos introduciéndolos en actividades despierten y fortalecen ciertos impulsos, que tienen ciertos propósitos y provoca ciertas consecuencias".

El medio social también es una base primordial para educarnos entre todos, ya que a medida que el individuo va evolucionando, va adquiriendo nuevas conductas y saberes, por ello el entorno familiar y la escuela como educadores de conocimiento, deben brindar en la persona un espacio pedagógico y tener presente que no se puede homogenizar como tradicionalmente ocurre, ya que cada persona piensa y percibe las cosas desde su propia postura y experiencia, se debe entablar una continua comunicación donde se deja ver al desnudo una continua disposición por parte del alumno que quiere aprender lo que desea, , lo que considera necesaria, ya que él influye que le ayudara a reconocer y así mismo potencializar sus saberes y sus propias capacidades.

Se puede decir que la reflexión de el papel del docente hacia el proceso escolar del alumno desde distintos paradigmas tanto humanista, cognitivo, político y ético, es un actuar a diario en el aula de clase, con una clara visión el cual es formar a los futuros profesionales de la Educacion Fisica, Recreacion y Deportes, desde un acto pedagógico que pasa a centrarse en la potencialización de los procesos de los estudiantes, de sus capacidades y destrezas que les permita en un futuro próximo, no solo comprender sino enfrentar de manera optima su ejercicio laboral.

Los modelos pedagógicos en Educacion Fisica, Recreacion y Deportes debe permitir a los nuevos docentes aportar a nuevos diseños de organización e implementación del currículo, y a las nuevas políticas educativas, para que así mismo como docentes comprendamos y valoremos el rico conjunto de necesidades y diferencias individuales que representan los alumnos, es por ello que el docente debe educar ciudadanos críticos productivos recordando sus derechos y deberes, siendo ejemplo cada vez hacia un programa académico con acreditación de alta calidad, que no solo se dedica en despertar el interés junto al conocimiento, sino que su objetivo también sea el de ampliar hacia nuevas tecnologías de la investigación y las ciencias para construir un país mejor.

Algunos especialistas en el campo de la Didáctica de la Educación Física argumentan la necesidad de este cuerpo de conocimientos "especial" por las diferencias sustanciales que observan entre la enseñanza y el aprendizaje que se producen comúnmente en el aula y los que 
se producen en el patio. Tales argumentaciones sostienen que el contexto físico es una variable importante dentro de los procesos de enseñanza aprendizaje y por ende, el principal factor de la necesidad de construir nuevas teorías sobre la enseñanza que en éste ámbito se producen.

Fernando Sánchez Bañuelos lo explica de este modo:

"La didáctica específica de la educación física y el deporte tiene que estar adaptada al desarrollo de una actividad de enseñanza en la que el movimiento corporal y el esfuerzo físico constituyen los contenidos. Pero en el caso del ámbito de la enseñanza de la educación física y el deporte las diferencias de los planteamientos didácticos van más allá de la peculiaridad de esta materia, extendiéndose a aspectos de fondo. Las características y requisitos de la enseñanza en el aula y las de nuestro ámbito son tan distintas que ésta plantea la necesidad de una didáctica especifica perfectamente diferenciada."

A partir de este planteo el autor expone una serie de diferencias entre la enseñanza de la educación física y el deporte y la enseñanza en el aula y agrega:

..."Estas diferencias consideradas en conjunto parecen llevarnos a la conclusión de que las clases de Educación Física y Deporte tienen un efecto más directo $e$ inmediato sobre el concepto que de sí mismo tienen los alumnos y también del profesor. La situación de clase, cuando los contenidos son relativos a la actividad física es más abierta y rica en posibilidades de relación interpersonal, con un posible efecto sobre el individuo de tipo más integral. Por esto mismo, los planteamientos didácticos se hacen más complejos, cuando las metas educativas y de enseñanza son progresistas y ambiciosas, la formación específica del docente de esta materia en cuanto a didáctica, tiene que ser más exigente que para el profesor de aula."
La necesidad de una didáctica "especial" parte más bien de lo "que" se enseña que del "ámbito" en que se enseña, aunque el que no pueda pensarse sin un contexto situacional en este caso. Es el contenido su aspecto de fondo y es la singularidad del mismo lo que configura a las llamadas "didácticas especiales". En ningún caso esta singularidad o especificidad puede pensarse como factor determinante del nivel de construcción teórica acerca de su enseñanza.

Ahora bien, en el caso de la Educación Física, el "que" se enseña resulta bastante problemático de resolver. Más aún si se considera el "que" se estudia. Dice Daniel Feldman "Las didácticas especializadas evolucionan asumiendo que el objeto de conocimiento determina el proceso de comunicación", pero en el caso de la Educación Física el contenido escolar no es necesariamente "ese objeto de conocimiento", un producto de la investigación teórica. El contenido escolar es una práctica social y cultural susceptible de ser estudiada teóricamente por lo tanto, es ésta práctica social y cultural la que determina su proceso de comunicación.

T. Popkewitz (1987) entiende el concepto de contenido como "una construcción social sin significado estático ni universal". Lo que en un momento determinado se consideran contenidos legítimos del currículum no puede extrapolarse a otros momentos de la historia de la Educación Física (ni al tiempo pasado ni al tiempo futuro) ni a otros lugares (culturas).

La concepción del sujeto que aprende como un cuerpo de similares características a la máquina ${ }^{3}$ en sus distintos tipos, ha dominado históricamente el campo de la Educación Física y ha sido la variable principal en la selección del contenido a enseñar. En un análisis histórico, la concepción de este cuerpo objetivado aparecía unida a la sumisión y dependencia 
de planteos disciplinares cuyas formas de producción del conocimiento se basaban en la experimentación y en los métodos hipotéticos deductivos. Resultado de esta dependencia ha sido la ausencia de explicaciones teóricas sobre la enseñanza de la educación física.

El cuerpo considerado como una máquina simple, como un sistema de fuerzas y palancas dominó el ámbito de la actividad física hasta los años cincuenta (Lagardera). La sumisión de los contenidos de la Educación Física a la Anatomía dio el marco epistemológico, cuyo objeto de conocimiento no podía ser otro que el movimiento de los músculos y huesos. Es por ello que en el campo escolar se priorizaron contenidos de orden biomecánico, y en la formación de profesores la Didáctica como teoría de la enseñanza estuvo ausente. Una visión empírica de la Educación Física con pretensiones científicas se ve reflejada en las prácticas de la época."

De aquí se entiende que los ejercicios físicos constituyeran un conjunto de posturas, con sus respectivos ángulos y palancas en la consideración de cada movimiento.

La concepción de cuerpo como máquina generadora de energía adquirió primacía cuando en la base de los programas escolares el contenido biomecánico buscó intencionalmente el desarrollo de los sistemas energéticos, musculares y cardiorrespiratorios. La sumisión de conocimientos necesarios para la fundamentación de la Educación Física, estaba ahora en manos de la Fisiología, la Termodinámica y la Psicología de tipo experimental.

El contenido escolar, de tipo "bioenergético" pretendía desarrollar capacidades fisiológicas y psicológicas, tal como observamos en los libros de texto utilizados en esa época:
"El concepto científico de la clase racional de ejercicios físicos según el sistema argentino es el siguiente: Producir en los alumnos, por medio de ejercicios físicos apropiados, una serie de efectos fisiológicos y psicológicos internos, graduados y suficientes para satisfacer los fines fundamentales de la educación física racional en su acepción escolar"7

La dependencia a planteos de campos diferentes como la Fisiología y la Psicología caracterizaron desde polos opuestos a las primeras explicaciones sobre la enseñanza de la Educación Física y algunas de las prácticas que engendraron perduran hasta nuestros días produciendo profundos debates sobre el qué y el cómo enseñar.

Bajo la sumisión de la Fisiología, la Educación Física sólo tiene un papel funcional ligado al desarrollo de las capacidades motrices: más fuerza, más resistencia, más velocidad. Los criterios de valoración de la acción educativa se centran en la eficacia y la eficiencia. El citius, altius, fortius, más lejos, más alto, más fuerte parece ser la consigna que define mejor estas explicaciones de enseñanza, reducidas a una teoría acerca de cómo ayudar en las clases a que los alumnos mejoren su condición física.

La tarea del docente se limita a programar instructivos en relación con las conductas observables y con la medición del rendimiento.

Bajo la sumisión de la Psicología, la Educación Física tiene un papel funcional ligado al desarrollo de capacidades psicológicas y aquí, la diversidad y la evolución de las distintas corrientes con sus grados de influencia merecen otro estudio aparte. Desde el desarrollo del carácter, la voluntad, la inteligencia, la creatividad y la autonomía, la enseñanza de la Educación Física parece lograr, según estos planteos, 
personas más equilibradas, más inteligentes, más autónomas o creativas.

El cuerpo considerado como máquina es sinónimo de visiones dualistas arrastradas desde los idearios griegos de "mens sana in corpore sano":

"El deporte y los ejercicios físicos ya no han de ser una válvula de escape para la alegría juvenil y la energía vital acumulada sino que deben ejercitar al cuerpo, hacerlo dócil instrumento de la voluntad..." Anemarie Seybold y Brunnhuber

La llamada Didáctica de la Educación Física nace en los programas de estudio del profesorado bajo la dependencia de estos planteos y en sus primeros pasos de construcción teórica es impregnada por planteos neuropsicológicos y acompañada por otra forma de concebir el cuerpo: la de la máquina informacional. El movimiento, que es generado por medio de un proceso de tratamiento de la información y el empleo del sistema nervioso, necesita adiestrarse, "educarse". Las disciplinas de base que sirven a estos planteos son la Cibernética y la Neuropsicología.

"...el modelo de la didáctica, basado en la teoría del aprendizaje, propone metas de estudio que han de asegurar al hombre el mejor equipamiento individual posible. La didáctica de orientación cibernética trata de racionalizar el proceso del aprendizaje y de perfeccionarlo por medio de los reconocimientos de la informática y la psicología de la información"(Seybold, 1976)

Esta influencia se ve acrecentada hacia los años 80 por las corrientes psicomotrices francesas y del motor learning americano.

El extremadamente complejo sistema nervioso, desde las áreas corticales superiores hasta las terminaciones sensoriales periféricas y nervios motores, con sus innumerables mecanismos de entrada y salida, desafía un análisis completo, aun cuando se posean algunos conocimientos acerca de sus sistema de facilitación propioceptiva, con una realimentación sensorial continua, de los servomecanismos de la médula $y$ mesoencéfalo y de las extensas áreas de asociación sensoriales, motrices y del cerebro mismo."

Este modelo explicativo sobre la educabilidad y enseñabilidad avanzó analógicamente a la evolución de las máquinas electrónicas. De máquinas que deciden en forma binaria a máquinas que deciden sobre numerosas alternativas considerando otro tipo de información del medio.

Las teorías explicativas acerca de la enseñanza siguen priorizando la eficacia, pero con un rol mas activo en el sujeto, que ahora percibe, decide y ejecuta.

En la actualidad se intenta dejar de lado la concepción de cuerpo como máquina. Los actuales planteos epistemológicos reciben la influencia de la Psicología Cognitiva, de las Teorías de la Comunicación, la Psicolingüística y las Teorías Sociocríticas.

La concepción de cuerpo sujeto y la capacidad de reflexionar sobre las acciones y otorgarle significados, permite pensar en otro tipo de saberes y en otros procesos de comunicación.

En la actualidad el desplazamiento de las concepciones ontológicas, epistemológicas y metodológicas nos lleva a reformular nuevamente las teorías sobre la enseñanza de la Educación Física. La concepción de sujeto corporal en vez de cuerpo objeto, la búsqueda de un objeto de estudio desde otros paradigmas y las investigaciones basadas en una pluralidad de métodos nos brindan un panorama muy distinto respecto a la enseñanza. 
Explicar hoy qué se enseña y cómo es reducir la Didáctica a una mera teoría instructiva. Entender lo social y cultural de nuestros saberes significativos es comprender su enseñanza en nuevos contextos con nuevos significados. Esto es lo que nos permite transformar la enseñanza bajo nuevas concepciones.

Pero ¿se trata de nuevas didácticas y nuevos contenidos o más bien se trata de un nuevo enfoque? ¿Puede haber una nueva didáctica cuando sus planteos teóricos siguen dependiendo del conocimiento de otras disciplinas?

La producción del conocimiento sobre la educabilidad y enseñabilidad de la Educación Física debe plantearse en la misma tarea de la enseñanza. Es en estas situaciones donde podemos hablar de un conocimiento "especial" . Sin embargo no podremos avanzar mucho en nuestro campo didáctico si no resolvemos las relaciones "especiales" que tenemos entre nuestro objeto de estudio y nuestro objeto de enseñanza.

\section{REFERENCIAS BIBLIOGRÁFICAS}

FERMOSO, Paciano. Teoría de la Educación, una interpretación antropológica. CEAC Ediciones S.A. 2da Edición. Barcelona, España. 1985. 365 pág.

Consejo Nacional de Acreditación. Bogotá, Colombia. Febrero, 2006.

ZAPATA, John y VÉLEZ, Lucía. Un 1er Acercamiento a la Investigación AcciónEducativa en la Formación de Maestros. En revista Investigando-nos. No. 1. Grupo CEDINEP-Colciencias. Fondo Editorial Biogénesis. Barranquilla, 2004.

ECHEVERRI, Guillermo. En revista Texto. No. 8. Pontificia Universidad Bolivariana.
Facultad de Educación. Pub. Semestral. Enero-Junio, 2004. Medellín. 